\title{
EFECTOS TERAPÉUticos POSITIVOS A TRAVÉS DEL AUMENTO DEL OPTIMISMO
}

\author{
POSITIVE THERAPEUTIC EFFECTS THROUGH \\ THE INCREASE OF OPTIMISM
}

\author{
Andrés Fernando López Pell \\ Alexis Kasanzew \\ Mariana Beatriz López \\ Universidad Católica de Santa Fe (UCSF) y FunSALED - Argentina
}

\begin{abstract}
Resumen: El optimismo parece ser un constructo significativo en cuanto permitiría predecir variables de notable importancia clínica. La psicología positiva plantea que el incremento del optimismo influye de manera significativa en la disminución de la sintomatología psicopatológica. De ser esto así, se podrían optimizar los efectos de la psicoterapia mediante un plan para incrementar el optimismo. Con el objetivo de probar esta hipótesis, se diseñó un estudio experimental con pacientes que recibían psicoterapia cognitiva clásica. Para evaluar cambios en los síntomas psicopatológicos se utilizó la escala SCL-90-R. Los resultados mostraron una disminución significativa en variables como somatizaciones, ansiedad fóbica y hostilidad a favor del grupo experimental en comparación con el grupo control. Por lo tanto, este plan para aumentar el optimismo parece producir una optimización de la psicoterapia cognitiva al disminuir la intensidad de los síntomas en los pacientes.
\end{abstract}

Palabras clave: optimismo, psicoterapia cognitiva, optimización

\begin{abstract}
Optimism seems to be a significant construct as it would predict variables of remarkable clinical importance. Positive psychology argues that the improvement of optimism has a significant influence in the decrease of psychopathological symptoms. Of being this thus, the effects of the psychotherapy could be optimized by means of a plan to increase optimism. With the aim of proving this hypothesis, an experimental study with patients who received classic mental psychotherapy was designed. In order to evaluate changes in the psychopathological symptoms, the SCL-90-R scale was used. The results showed a significant decrease in variables such as somatizations, phobic anxiety and hostility in favor of the experimental group in comparison with the control group. Therefore, this plan to increase optimism seems to produce an optimization of the cognitive psychotherapy when decreasing the symptoms intensity of the patients.
\end{abstract}

Key words: optimism, cognitive psychotherapy, optimization

Correspondencia: Prof. Andrés López Pell. Licenciado en Psicología. Universidad Católica de Santa Fe. Facultad de Psicología, Argentina. Correo Electrónico: alopezpell@funsaled.org.ar. 


\section{INTRODUCCIÓN}

Aunque conceptos tales como pensamiento positivo u optimismo han formado parte de lo que podríamos denominar psicología popular desde hace ya muchos años, solo recientemente han empezado a ser objeto de investigación rigurosa en el campo de la psicología. A pesar de este inicio tardío, la cantidad de información generada en relación con el tema en las dos últimas décadas ha sido muy importante. El interés por el constructo optimismo no se debe tanto a preocupaciones teóricas, como al hecho de que el mismo permitiría predecir variables de notable importancia clínica. Scheier y Carver (1985) definen tanto al optimismo como al pesimismo como expectativas generalizadas acerca de las cosas que le suceden a uno en la vida. Tales expectativas se consideran además como disposiciones estables o rasgos de personalidad. Cabría preguntarse entonces: ¿estos estilos diferentes de afrontamiento influyen en la calidad de vida y en la salud mental? De acuerdo con el modelo de autorregulación de la conducta postulado por los autores antes mencionados, las personas con expectativas positivas en relación con el futuro -los optimistas- tenderán a prolongar sus esfuerzos hacia las metas, aún cuando tengan que enfrentar serios obstáculos. Por el contrario, las personas con poca confianza -los pesimistas- serán más propensas a evitar la adversidad, realizando actividades distractivas temporarias que no ayudan a resolver el problema. Estos esfuerzos por enfrentar o evitar los problemas se traducen en diferentes comportamientos, que constituyen lo que se denomina estrategias de afrontamiento.

El hecho de que los optimistas experimenten menos estrés que los pesimistas al enfrentarse con dificultades en su vida podría deberse al modo particular en que las afrontan, lo cual fomenta una mejor adaptación. En este sentido, los optimistas utilizan más estrategias de afrontamiento centradas en el problema, así como una variedad de estrategias de afrontamiento centradas en emociones positivas, además de trabajar para aceptar la realidad de situaciones difíciles y mirar los acontecimientos de la mejor manera posible (Carver, Scheier \& Weintraub, 1989; Fontaine, Manstead \& Wagner, 1993; Libram, 2002; Scheier, Weintraub \& Carver, 1986). Por su parte, los pesimistas tienden a focalizar en sus emociones negativas, en el distanciamiento mental y la negación de la realidad (Boland \& Coppeliez, 1997). Revelan una tendencia a desentenderse de las metas con las cuales interfiere un estresor, así como al abuso de substancias y hacia otras conductas que contribuyen a disminuir el nivel de conciencia en relación con el problema (Carver, Scheier \& Weintraub, 1989; Fontaine, Manstead \& Wagner, 1993).

La relación entre optimismo y estrés fue examinada en diversos grupos de personas que enfrentaban situaciones difíciles o adversidad, intentando, a través de múltiples mediciones, determinar si los pesimistas experimentan más estrés que los optimistas antes y después de estos acontecimientos.

El optimismo se halló en todos los casos relacionado con niveles más bajos de estrés al enfrentar los estresores, aún controlando el nivel del mismo previo al evento adverso (e.g., Aspinwall \& Taylor, 1992; Carver \& Gaines, 1987; Carver \& Scheier, 2002; Carver et al., 1993; Cozzarelli, 1993; Given et al., 1993; Hooker Monahan, Shifren \& Hutchinson, 1992; Litt, Tennen, Affleck, \& Klock, 1992; Scheier et al., 1989; Fitzgerald et al., 1993; Shifren \& Hooker, 1995).

Por todo lo expuesto se nota que existe amplio consenso en la comunidad académica en torno a los beneficios del modo optimista de pensar. Es evidente que este proceso está muy ligado a la forma en que las personas connotan los sucesos de la vida. Partiendo de esta base se podría hipotetizar que haciendo más positiva la manera de connotar (i.e., orientándola optimistamente) se podría lograr una disminución de sintomatología psicopatológica.

Con respecto a la posible disminución de sintomatología psicopatológica hay variados dispositivos psicoterapéuticos, entre ellos la psicoterapia cognitiva, que han demostrado ser eficaces en este sentido (para una mayor ampliación véase, Liddon \& Jones, 2002), pero la pregunta que se intenta responder en este trabajo es si ¿se puede optimizar los efectos de la psicoterapia cognitiva mediante el agregado de intervenciones destinadas a incrementar el optimismo? Para tratar de corroborar esta hipótesis es que se intento lograr esta optimización mediante la utilización del modelo ACCRR ideado por Seligman (2003). Se trata de un plan que actúa directamente la manera de connotar de las personas. Lamentablemente hasta la actualidad los autores de este trabajo desconocemos 
la existencia de estudios de este tipo y con esta finalidad tan específica. A continuación se presentan los resultados experimentales de como se logró poner a prueba la hipótesis planteada.

\section{OBJETIVOS}

El presente estudio se propuso como objetivo verificar si la psicoterapia puede verse favorecida a través del incremento del optimismo en el paciente, a partir de la hipótesis que el mejoramiento del optimismo influye positivamente en la disminución de sintomatología psicopatológica. Para tal efecto, se aplicó el plan de mejoramiento del optimismo ACCRR (i.e., A $=$ Adversidad, $\mathrm{C}=$ Creencias o ideas previas, $\mathrm{C}$ = Consecuencias habituales de dichas ideas, $\mathrm{R}$ = Rebatimiento de la creencias originaria, y $R=$ Revitalización, cuando la creencia es refutada con éxito) ideado por Seligman (2003), y se evaluaron síntomas psicopatológicos a través de la escala SCL-90-R de Derogatis (1994).

\section{MÉTODO}

\section{Muestra}

Participaron en total 60 pacientes de gravedad media a baja, quienes se incluyeron en el estudio a medida que ingresaban al servicio de salud mental de FUNSALED, institución dedicada a la atención psicológica en la ciudad de Florida, Provincia de Buenos Aires. La media de edad fue de 34,71 (d.t. 11,53), siendo para el grupo control de 31,76 (d.t. 10,25) y para el experimental de 37,66 (d.t. 12,15). En cuanto al sexo, 19 participantes eran varones $(31,6 \%)$ y 41 mujeres $(68,4 \%)$. Por otro lado, 23 sujetos $(38,3 \%)$ vivían en la Ciudad de Buenos Aires y $37(61,7 \%)$ en el conurbano bonaerense.

\section{Diseño}

Se trabajó con un diseño experimental. Los participantes fueron evaluados con el instrumento SCL-90-R en su versión adaptada para población argentina (Casullo, 2004), quienes fueron asignados alternativamente por azar simple al grupo control y al grupo experimental. Ambos grupos participaron de sesiones de psicoterapia cognitiva clásica, entendida como un sistema de psicoterapia basado en las teorías sobre el procesamiento patológico de la información en los trastornos mentales. El tratamiento se dirige esencialmente a modificar las cogniciones distorsionadas o desadaptativas y las alteraciones conductuales que conllevan. EI énfasis se pone en problemas específicos 0 cuestiones de la vida actual del paciente porque estimula la elaboración de planes de acción que pueden ayudar a contrarrestar síntomas disfuncionales. El objetivo es lograr una valoración precisa y objetiva de la validez de las cogniciones y la naturaleza adaptativa de las conductas frente a las desadaptativas. Si se producen distorsiones cognitivas, el paciente y el terapeuta trabajan juntos para desarrollar una perspectiva más racional (i.e. empirismo colaborativo). Por otra parte, si se identifican experiencias o características actuales negativas, se intentará encontrar formas de afrontarlas o de cambiarlas (para una revisión más amplia véase Beck, Rush, Shaw y Emery, 1983; Ellis y Grieger, 1981).

No obstante, en el grupo experimental se dedicaron cinco minutos de cada sesión a la aplicación del protocolo ACCRR. La duración total de las sesiones de ambos grupos fue de aproximadamente 40 minutos y las mismas fueron llevadas a cabo por varios terapeutas de FUNSALED, con una formación profesional homogénea. Luego de cinco sesiones, los integrantes de ambos grupos fueron evaluados nuevamente en un re-test general con la SCL-90-R.

La aplicación del protocolo ACCRR consistió en los siguientes pasos secuenciales: a) explicar al paciente el modelo de mediación cognitiva según el cual nuestras emociones y conductas dependen de las creencias que se tienen acerca de las cosas y de sí mismos; b) Hablar sobre las creencias disfuncionales que resultan un obstáculo para la obtención de metas deseadas; c) Identificar con el paciente alguna creencia pesimista y enseñarle cómo analizarla, instruyéndolo en las cuatro maneras propuestas por Seligman (2003) para discutir con uno mismo: evidencia, alternativas, implicaciones, y utilidad; $y, d$ ) pedirle al paciente que durante la semana siguiente se muestre receptivo a su dialogo interno ante la más mínima adversidad, aplicando lo aprendido en la sesión. Con el fin de hacer más significativo el ejercicio, tal como aconseja Seligman (2003), se le pidió al paciente que lleve un registro de todo el procedimiento ajustándose al siguiente formato $A C C R R$.

En las sesiones posteriores se revisaba el trabajo realizado por el paciente y corregían los 
errores que pudieran aparecer tanto en la comprensión del ejercicio como en su lógica. Se colaboró con el sujeto en la discusión de las creencias y se le pidió que repita la tarea en las semanas siguientes siguiendo el mismo procedimiento.

\section{Instrumento}

Para la evaluación de los síntomas psicopatológicos se eligió la escala SCL-90 diseñada por Derogatis (1994), y se aplicó la adaptación para población argentina (i.e., SCL90-R) realizada por Casullo (2004). Esta escala evalúa patrones de síntomas que el sujeto entrevistado experimenta en el presente o ha vivenciado durante la última semana. Las respuestas son evaluadas en función de nueve dimensiones primarias: 1) Somatizaciones: evalúa la presencia de malestares relacionados con diferentes disfunciones corporales; 2) Obsesiones y compulsiones: identifica pensamientos, acciones e impulsos que son vivenciados como imposibles de evitar o no deseados; 3) Sensitividad Interpersonal: detecta la presencia de sentimientos de inferioridad e inadecuación, en especial cuando la persona se compara con sus semejantes; 4) Depresión: representa manifestaciones clínicas como el estado de ánimo disfórico, la falta de motivación, la poca energía vital, los sentimientos de desesperanza y la ideación suicida; 5) Ansiedad: evalúa la presencia de nerviosismo, tensión, ataques de pánico y miedos; 6) Hostilidad: esta dimensión hace referencia a pensamientos, sentimientos y acciones característicos de la presencia de afectos negativos de enojo; 7) Ansiedad fóbica: alude a una respuesta persistente de miedo a personas específicas, lugares, objetos o situaciones que es en sí misma irracional y desproporcionada en relación con el estímulo que la provoca; 8) Ideación paranoide: evalúa comportamientos como el pensamiento proyectivo, la suspicacia y el temor a la pérdida de autonomía; y 9) Psicoticismo: incluye síntomas relacionados con sentimientos de soledad, estilo de vida esquizoide, alucinaciones y control del pensamiento. Asimismo, cuenta con tres índices globales de malestar psicológico: a) Índice de Severidad Global (ISG), que muestra el nivel actual de la severidad del malestar; b) Total de síntomas positivos (STP), que refleja un intento consciente de la persona de mostrarse mejor de lo que realmente está; y c) Índice de Malestar Positivo (PSDI), que pretende evaluar el estilo de respuesta indicando si la persona tiende a exagerar o a minimizar los malestares que la aquejan.

\section{RESULTADOS}

Para el análisis de los resultados se realizó un análisis de varianza $2 \times 2$, considerando como factores intersujetos el grupo experimental (Control vs. Experimental), y como factor intrasujeto el tiempo anterior y posterior al tratamiento. Las medias y desvíos estándar para cada grupo antes y después de 5 sesiones de tratamiento se informan en la Tabla 1. En este trabajo se muestran las seis sub escalas y los dos índices globales de malestar psicológico de la SCL-90$\mathrm{R}$ que arrojaron diferencias significativas entre los grupos comparados antes y después de la quinta sesión del tratamiento. Las demás dimensiones (i.e., obsesiones y compulsiones, sensitividad interpersonal, psicoticismo e índice de malestar positivo) no mostraron diferencias estadísticamente significativas.

Somatizaciones. Como se observa en el gráfico 1, el análisis de varianza mostró efectos de interacción entre el tiempo anterior o posterior del tratamiento y los grupos experimentales, $F_{(1,58)}=8,535 ; M S E=2,067 ; p<0,01$. El análisis de los efectos simples muestra que el grupo control, mostró diferencias en sus mediciones antes y después del tratamiento, éste grupo puntuó significativamente más bajo después del tratamiento que antes de él, $t_{(29)}=2,588$; $p<0,05$. Asimismo, el grupo Experimental puntuó significativamente más bajo después del tratamiento que antes de él, $t_{(29)}=5,130$; $p<0,001$. La diferencia en ambos grupos radica en que en el grupo Experimental la diferencia fue significativamente mayor que en el grupo control, potenciándose este efecto en éste grupo, $t_{(58)}=2,921 ; p<0,01$ (ver Gráfico 1).

Sensibilidad interpersonal. Como se observa en el gráfico 2 el análisis de varianza no mostró efectos de interacción entre el tiempo anterior o posterior del tratamiento y los grupos experimentales, $F_{(1,58)}=0,256 ; M S E=5,512 ; p$ $=0,615$. El Análisis mostró diferencias por efecto del tiempo anterior y posterior al tratamiento para ambos grupos, que disminuyeron significativamente en sus puntajes, $F_{(1,58)}=$ 104,$577 ;$ MSE $=5,512 ; p<0,001$. Pero se observaron diferencias entre los grupos 
Tabla 1: Medias y desvíos estándar por grupo, antes y después del tratamiento

\begin{tabular}{|c|c|c|c|c|c|}
\hline $\begin{array}{l}\text { Subescalas } \\
\text { SCL-90 R }\end{array}$ & Grupos & $\begin{array}{c}\text { Medlas } \\
\text { antes }\end{array}$ & $\begin{array}{l}\text { Desv. } \\
\text { tip. } \\
\text { Antes }\end{array}$ & $\begin{array}{l}\text { Medlas } \\
\text { despues }\end{array}$ & $\begin{array}{c}\text { Desv. } \\
\text { tip. } \\
\text { Después }\end{array}$ \\
\hline \multirow{2}{*}{ Somatizaciones } & Control & 3,2 & 1,627 & 2,47 & 0,507 \\
\hline & Experimental & 4,87 & 2,161 & 2,6 & 1,38 \\
\hline \multirow{2}{*}{ Obsesiones } & Control & 19,17 & 3,196 & 15,87 & 3,748 \\
\hline & Experimental & 19,07 & 3.903 & 17 & 3,04 \\
\hline \multirow{2}{*}{$\begin{array}{l}\text { Sensibilidad } \\
\text { interpersonal }\end{array}$} & Control & 17,37 & 3,828 & 13,2 & 2,441 \\
\hline & Experimental & 19.27 & 3.393 & 14,67 & 1.322 \\
\hline \multirow{2}{*}{ Depresión } & Control & 25,43 & 4,651 & 18,73 & 3,226 \\
\hline & Experimental & 25.53 & 6,399 & 20 & 3.88 \\
\hline \multirow{2}{*}{ Ansiedad } & Control & 16,17 & 4,8 & 10,43 & 2,944 \\
\hline & Experimental & 15,87 & 4,297 & 8,87 & 2,776 \\
\hline \multirow{2}{*}{ Hostilidad } & Control & 10,07 & 1,484 & 7 & 2,533 \\
\hline & Experimental & 11,27 & 2,243 & 6,6 & 1,773 \\
\hline Ansledad & Control & 5,97 & 1,974 & 3,63 & 1,45 \\
\hline fóbica & Experimental & 7,33 & 2,023 & 3,27 & 1,874 \\
\hline Ideación & Control & 7,83 & 3,217 & 5,37 & 2,385 \\
\hline paranoide & Experimental & 8,93 & 1,461 & 5,07 & 2,116 \\
\hline \multirow{2}{*}{ Psicoticismo } & Control & 10,5 & 2,933 & 10,57 & 2,063 \\
\hline & Experimental & 9,8 & 2,929 & 10,07 & 1,68 \\
\hline \multirow{2}{*}{$\begin{array}{l}\text { Índice de } \\
\text { severidad }\end{array}$} & Control & 1,2803 & 0,17547 & 0,9937 & 0,1321 \\
\hline & Experimental & 1,358 & 0.11932 & 0,952 & 0,10791 \\
\hline \multirow{2}{*}{$\begin{array}{l}\text { Global } \\
\text { Índice de } \\
\text { síntomas } \\
\text { positivos }\end{array}$} & Control & 58,13 & 4,416 & 52,73 & 4,291 \\
\hline & Experimental & 59,27 & 2,638 & 47,07 & 5,044 \\
\hline \multirow{2}{*}{$\begin{array}{l}\text { Indice de } \\
\text { malestar } \\
\text { positivo }\end{array}$} & Contról & 1,9841 & 0,17928 & 1,6968 & 0,10686 \\
\hline & Experimental & 2,0608 & 0,19721 & 1,8291 & 0,15343 \\
\hline
\end{tabular}

experimentales (Control vs. Experimental), $F_{(1,58)}$ $=7,441 ; M S E=11,424 ; p<0,01$. Estas diferencias observadas muestran que el grupo Experimental puntuó siempre tanto antes como después del tratamiento más alto que el grupo Control. Ésta diferencia no puede ser explicada por un efecto del tratamiento, sino a diferencias individuales debidas a características propia de los sujetos (ver Gráfico 2).

Depresión. El análisis de varianza no mostró efectos de interacción entre el tiempo anterior o posterior del tratamiento y los grupos experimentales, $F_{(1,58)}=3,033 ; M S E=17,146 ; p$ $=0,087$ (ver Gráfico 3). El Análisis mostró diferencias por efecto del tiempo anterior y posterior al tratamiento para ambos grupos, posterior al tratamiento los puntajes disminuyeron significativamente, $F_{(1,58)}=52,607 ; M S E=$ 17,$146 ; p<0,001$. No se mostraron efectos en la diferencia entre los grupos experimentales (Control vs. Experimental) $F_{(1,58)}=1,598 ; M S E$ $=27,795 ; p=0,211$ (ver Gráfico 3). 
Gráfico 1. Somatizaciones: varianza entre grupos según el tiempo anterior y posterior al tratamiento

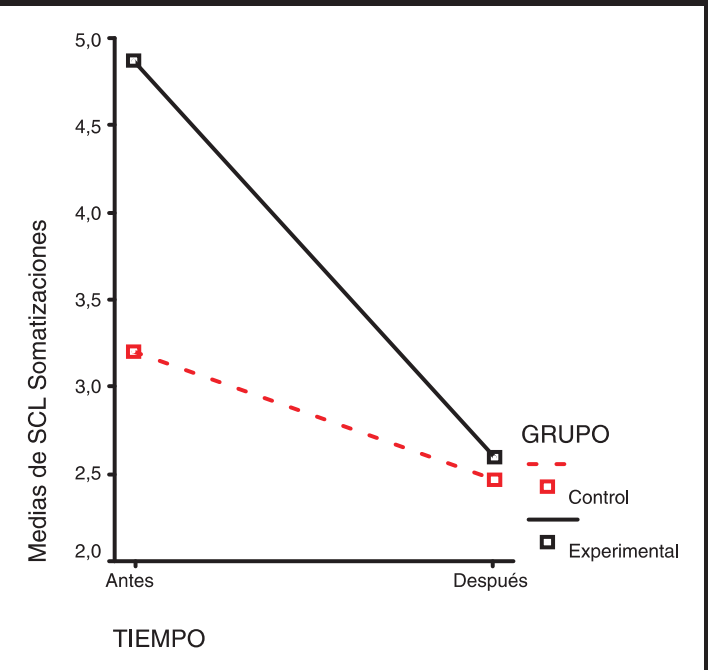

Ansiedad. El análisis de varianza no mostró efectos de interacción entre el tiempo anterior o posterior del tratamiento y los grupos experimentales, $F_{(1,58)}=1,206 ; M S E=9,982 ; p$ $=0,277$. El Análisis mostró diferencias por efecto del tiempo anterior y posterior al tratamiento para ambos grupos, posterior al tratamiento los puntajes disminuyeron significativamente, $F_{(1,58)}$ $=121,827 ; M S E=9,982 ; p<0,001$. $Y$ no se mostraron efectos en la diferencia entre los grupos experimentales (Control vs. Experimental) $F_{(1,58)}=1,379 ; M S E=18,957 ; p=0,245$ (ver Gráfico 4).

Hostilidad. El análisis de varianza mostró efectos de interacción entre el tiempo anterior o posterior del tratamiento y los grupos experimentales, $F_{(1,58)}=5,102 ; M S E=3,763 ; p$ $<0,05$ (ver Gráfico 5). El análisis de los efectos simples muestra que el grupo control, mostró diferencias en sus mediciones antes y después del tratamiento, éste grupo puntuó significativamente más bajo después del tratamiento que antes de él, $t_{(29)}=6,185 ; p<$ 0,001. Asimismo, el grupo Experimental puntuó significativamente más bajo después del tratamiento que antes de él, $t_{(29)}=9,224 ; p<$ 0,001 . La diferencia en ambos grupos radica en que en el grupo Experimental la diferencia fue significativamente mayor que en el grupo control, potenciándose este efecto en éste grupo, $t_{(58)}=2,259 ; p<0,05$. (ver Gráfico 5).
Gráfico 2. Sensibilidad interpersonal: varianza entre grupos según el tiempo anterior y posterior al tratamiento

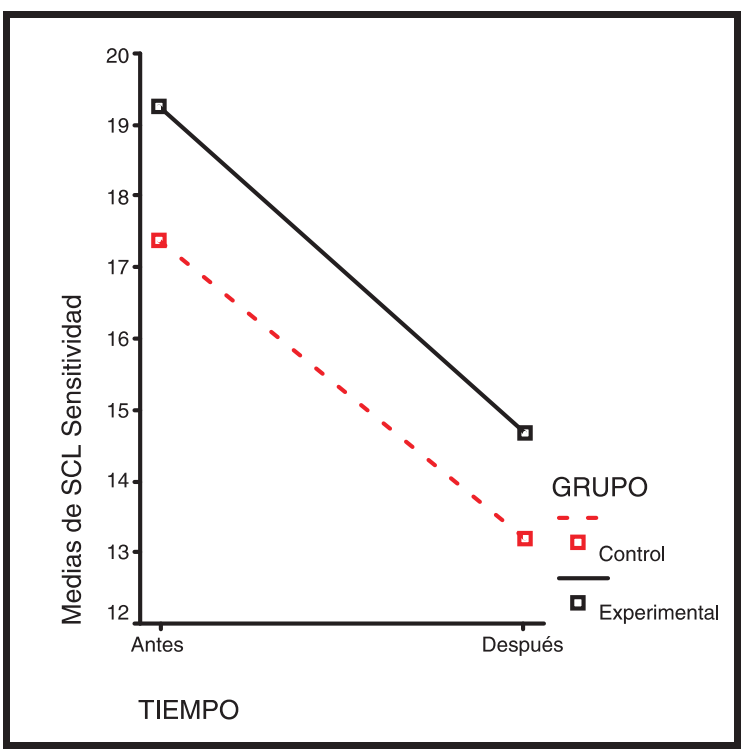

Gráfico 3. Depresión: varianza entre grupos según el tiempo anterior y posterior al tratamiento

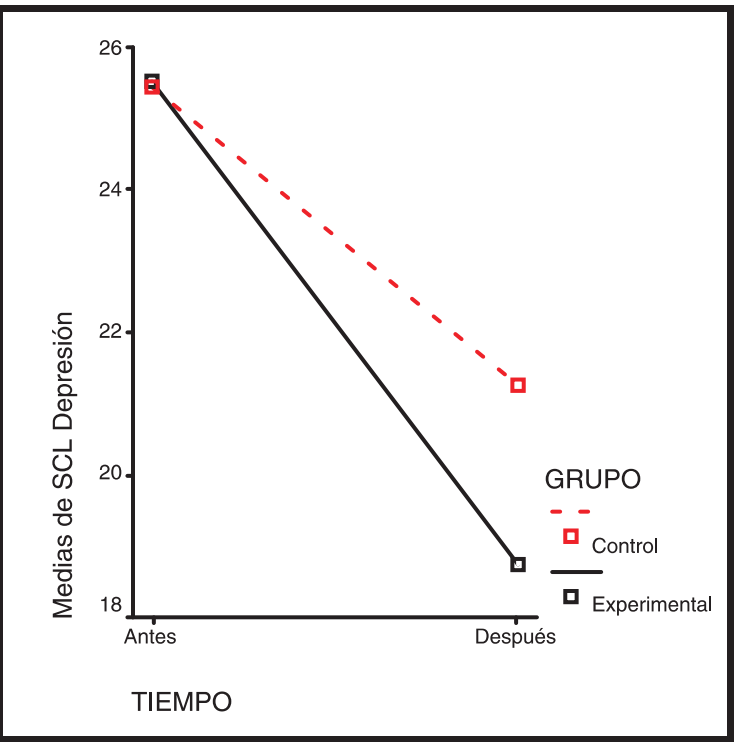

Ansiedad fóbica. El análisis de varianza mostró efectos de interacción entre el tiempo anterior o posterior del tratamiento y los grupos experimentales, $F_{(1,58)}=6,559 ;$ MSE $=3,436 ; p$ $<0,05$ (ver Gráfico 6). El análisis de los efectos simples muestra que el grupo control, mostró diferencias en sus mediciones antes y después del tratamiento, éste grupo puntuó significativamente más bajo después del tratamiento que antes de él, $t_{(29)}=4,551 ; p<$ 0,001 . Asimismo, el grupo Experimental puntuó 
Gráfico 4. Ansiedad: varianza entre grupos según el tiempo anterior y posterior al tratamiento

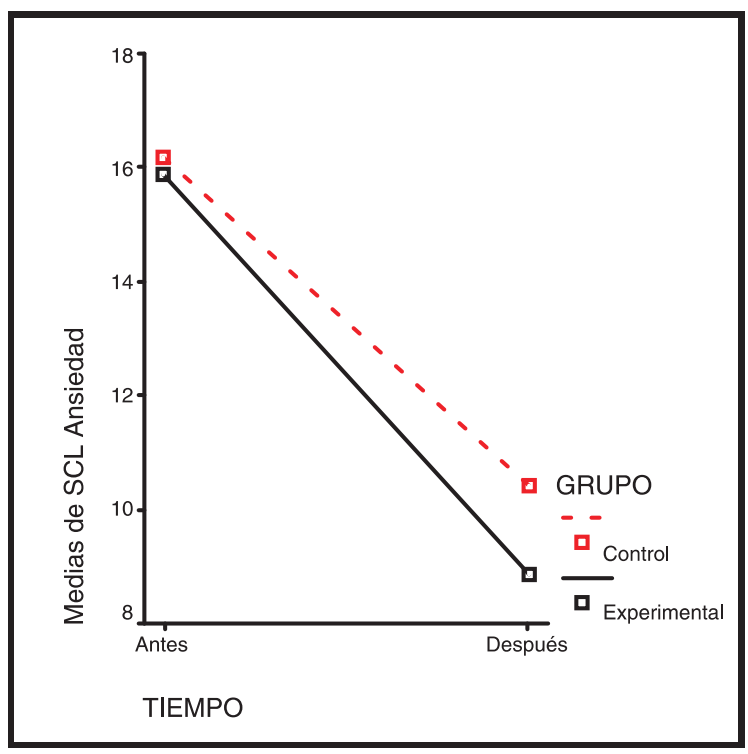

Gráfico 5. Hostilidad: varianza entre grupos según el tiempo anterior y posterior al tratamiento

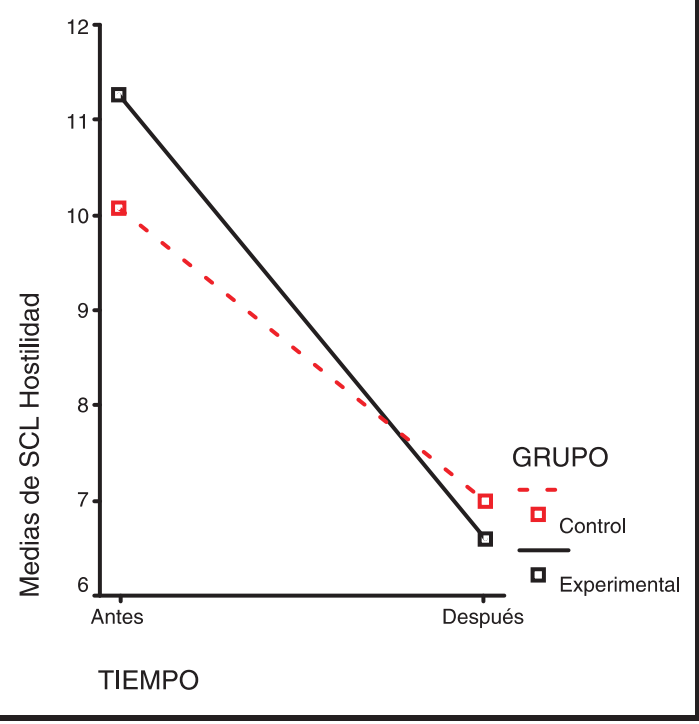

significativamente más bajo después del tratamiento que antes de él, $t_{(29)}=9,203 ; p<$ 0,001 . La diferencia en ambos grupos radica en que en el grupo Experimental la diferencia fue significativamente mayor que en el grupo control, potenciándose este efecto en éste grupo, $t_{(58)}=2,561 ; p<0,05$ (ver Gráfico 6).

Ideación paranoide. El análisis de varianza mostró efectos de interacción entre el tiempo anterior o posterior del tratamiento y los grupos experimentales, $F_{(1,58)}=4,500 ; M S E=3,267 ; p$ $<0,05$. El análisis de los efectos simples
Gráfico 6. Ansiedad fóbica: varianza entre grupos según el tiempo anterior y posterior al tratamiento

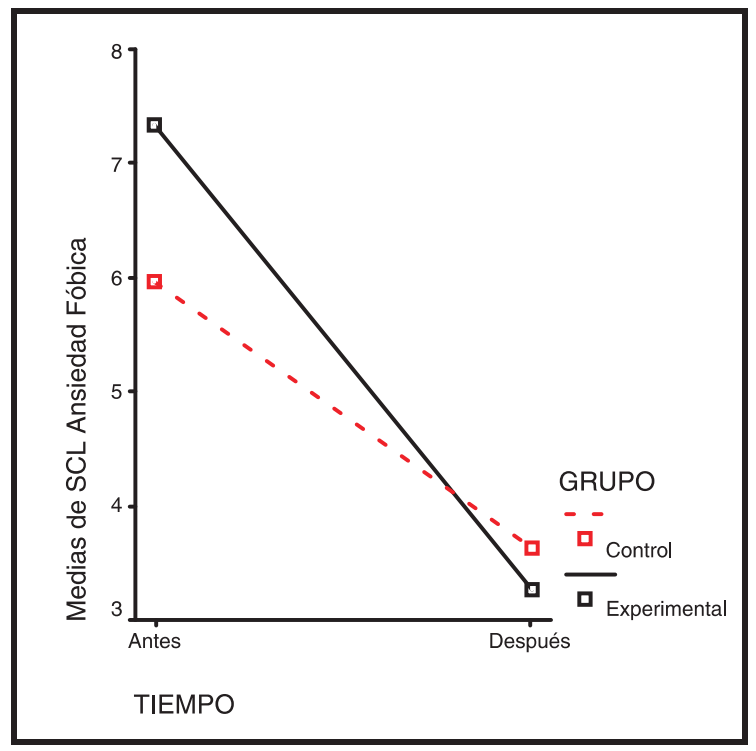

muestra que el grupo control, mostró diferencias en sus mediciones antes y después del tratamiento, éste grupo puntuó significativamente más bajo después del tratamiento que antes de él, $t_{(29)}=4,663 ; p<$ 0,001 . Asimismo, el grupo Experimental puntuó significativamente más bajo después del tratamiento que antes de él, $t_{(29)}=9,799 ; p<$ 0,001 . La diferencia en ambos grupos radica en que en el grupo Experimental la diferencia fue significativamente mayor que en el grupo control, potenciándose este efecto en éste grupo, $t_{(58)}=2,121 ; p<0,05$ (ver Gráfico 7 ).

Psicoticismo. El análisis de varianza no mostró efectos de interacción entre el tiempo anterior o posterior del tratamiento y los grupos experimentales, $F_{(1,58)}=0,064 ; M S E=4,705 ; p=$ 0,802 . El Análisis no mostró diferencias por efecto del tiempo anterior y posterior al tratamiento para ambos grupos, $F_{(1,58)}=0,177 ; M S E=4,705 ; p=$ 0,675 . Como tampoco se observan efectos en la diferencia entre los grupos experimentales (Control vs. Experimental) $F_{(1,58)}=1,454 ; M S E=$ 7,425; $p=0,233$ (ver Gráfico 8).

Índice de severidad global. El análisis de varianza mostró efectos de interacción entre el tiempo anterior o posterior del tratamiento y los grupos experimentales, $F_{(1,58)}=5,016 ; M S E=$ 0,$021 ; p<0,05$. El análisis de los efectos simples muestra que el grupo control, mostró diferencias en sus mediciones antes y después del tratamiento, éste grupo puntuó significativa- 
Gráfico 7. Ideación paranoide: varianza entre grupos según el tiempo anterior y posterior al tratamiento

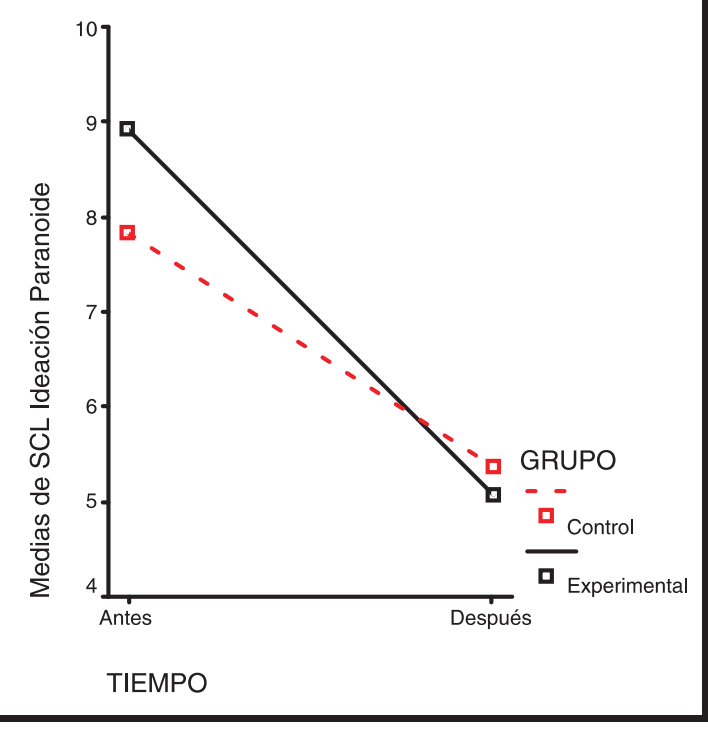

Gráfico 8. Psicoticismo: varianza entre grupos según el tiempo anterior y posterior al tratamiento

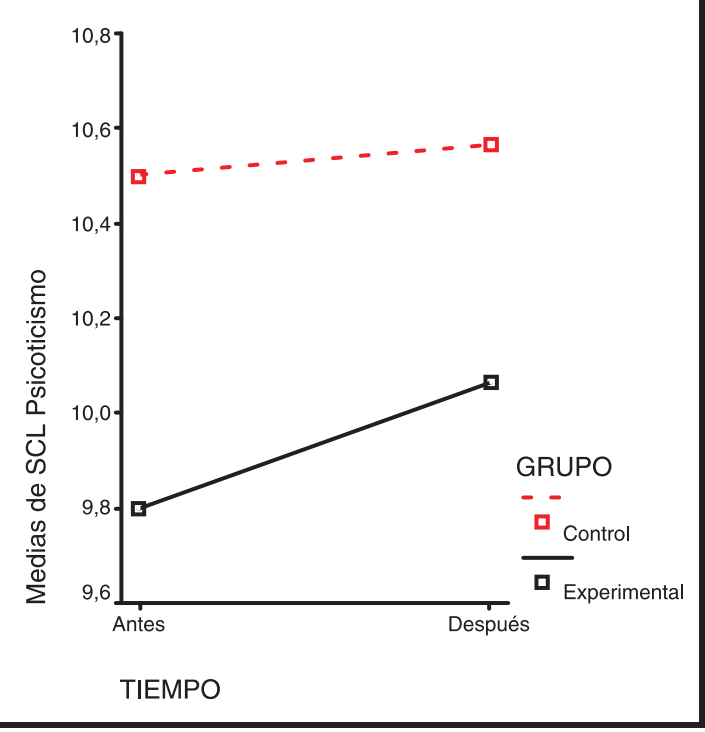

mente más bajo después del tratamiento que antes de él, $t_{(29)}=6,683 ; p<0,001$. Asimismo, el grupo Experimental puntuó significativamente más bajo después del tratamiento que antes de él, $t_{(29)}=12,831 ; p<0,001$. La diferencia en ambos grupos radica en que en el grupo Experimental la diferencia fue significativamente mayor que en el grupo control, potenciándose este efecto en éste grupo, $t_{(58)}=2,240 ; p<0,05$ (ver Gráfico 9).

Índice de síntomas positivos. El análisis de varianza mostró efectos de interacción entre
Gráfico 9. Índice de severidad global: varianza entre grupos según el tiempo anterior y posterior al tratamiento

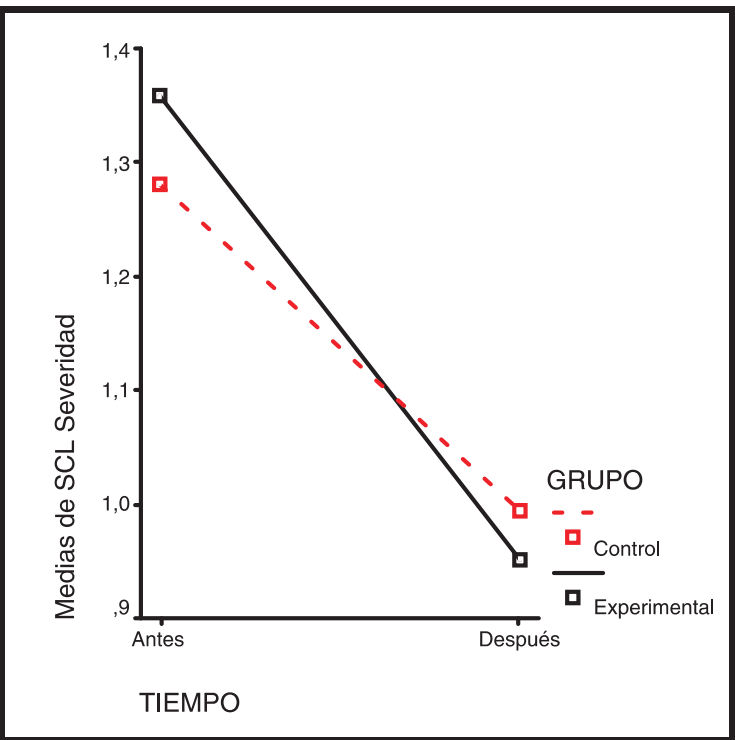

el tiempo anterior o posterior del tratamiento y los grupos experimentales, $F_{(1,58)}=19,548$; $M S E$ $=17,741 ; p<0,001$. El análisis de los efectos simples muestra que el grupo control, mostró diferencias en sus mediciones antes y después del tratamiento, éste grupo puntuó significativamente más bajo después del tratamiento que antes de él, $t_{(29)}=5,415 ; p<$ 0,001 . Asimismo, el grupo Experimental puntuó significativamente más bajo después del tratamiento que antes de él, $t_{(29)}=10,419 ; p<$ 0,001 . La diferencia en ambos grupos radica en que en el grupo Experimental la diferencia fue significativamente mayor que en el grupo control, potenciándose este efecto en éste grupo, $t_{(58)}=4,421 ; p<0,001$ (ver Gráfico 10).

Índice de malestar positivo. El análisis de varianza no mostró efectos de interacción entre el tiempo anterior o posterior del tratamiento y los grupos experimentales, $F_{(1,58)}=0,734 ; M S E$ $=0,032 ; p=0,395$. El Análisis mostró diferencias por efecto del tiempo anterior y posterior al tratamiento para ambos grupos, que disminuyeron significativamente en sus puntajes, $F_{(1,58)}=63,914 ;$ MSE $=0,032 ; p<$ 0,001 . Pero se observaron diferencias entre los grupos experimentales (Control vs. Experimental), $F_{(1,58)}=15,328 ; M S E=0,021 ; p<0,001$. Estas diferencias observadas muestran que el grupo Experimental puntuó siempre tanto antes como después del tratamiento más alto que 
Gráfico 10. Índice de síntomas positivos: varianza entre grupos según el tiempo anterior y posterior al tratamiento

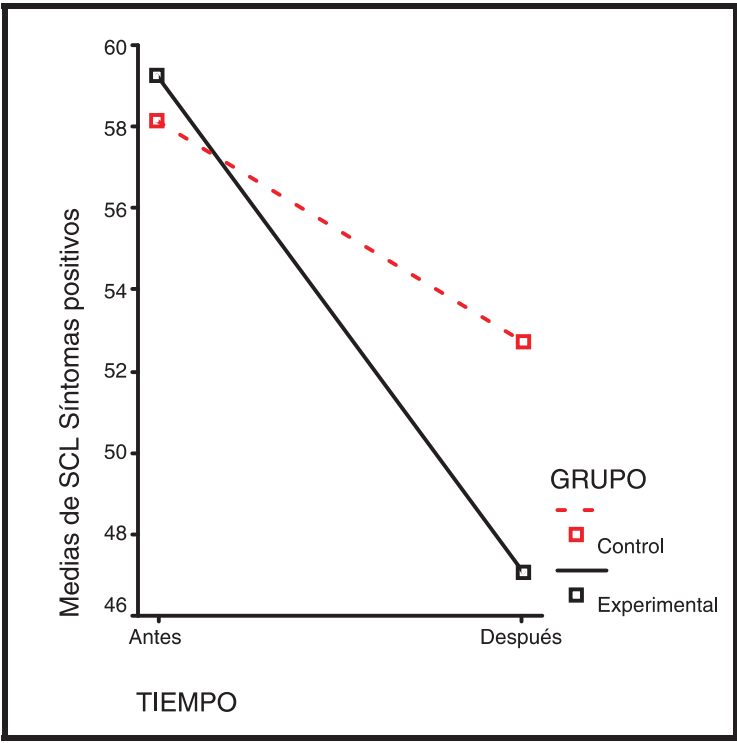

el grupo Control. Ésta diferencia no puede ser explicada por un efecto del tratamiento, sino a diferencias individuales debidas a características propia de los sujetos, o diferencias preexistentes al inicio tratamiento (ver Gráfico 11).

\section{DISCUSIÓN}

El modelo $A C C R R$ utilizado con el grupo experimental produjo diferencias estadísticamente significativas cuando se lo compara con el grupo control en la disminución de síntomas somáticos (i.e., malestares relacionados con diferentes disfunciones corporales), fóbicos (i.e., respuesta persistente de miedo hacia personas, lugares, objetos o situaciones) y hostiles (i.e., pensamientos, sentimientos y acciones que acompañan afectos negativos de enojo). Asimismo, los resultados muestran el beneficio de la técnica utilizada en los índices de severidad global y de síntomas positivos que ofrece el instrumento SCL-90-R. No obstante, el protocolo ACCRR no produjo cambios significativos en las escalas obsesiones y compulsiones, sensitividad interpersonal, psicoticismo e índice de malestar positivo.

Si bien estos resultados son muy alentadores se debe tener en cuenta que a nivel longitudinal solo se investigaron los efectos de la aplicación de este modelo durante cinco sesiones. Los procesos psicoterapéuticos son de una duración mucho mayor y restaría comprobar su influencia total a lo largo de todo un tratamiento.
Gráfico 11. Índice de malestar positivo: varianza entre grupos según el tiempo anterior y posterior al tratamiento

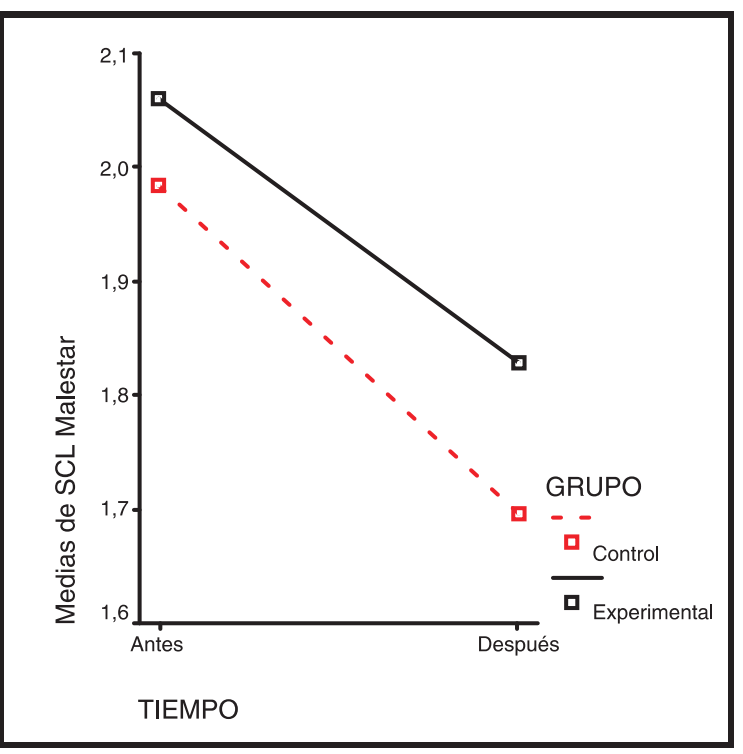

\section{CONCLUSIÓN}

Los resultados arrojados por la investigación verifican que la aplicación del modelo ACCRR en pacientes de baja y media gravedad logra una optimización y potenciación de los efectos de la psicoterapia cognitiva clásica en las primeras cinco sesiones al aumentar la visión optimista y disminuir la intensidad de los síntomas psicopatológicos en el paciente.

Este es un primer estudio clínico que, dada lo heterogeneidad de la muestra (i.e., pacientes clínicos de gravedad media a baja), necesitará repetirse en poblaciones más específicas (e.g., grupos de pacientes con trastornos de ansiedad o del estado de ánimo). Asimismo, estas posteriores investigaciones podrían incluir, por ejemplo, la evaluación de la relación entre las características de personalidad de los pacientes y su incidencia en los cambios sintomatológicos registrados por la SCL-90-R.

Otra cuestión de suma importancia sería la posibilidad de realizar un seguimiento longitudinal más amplio (i.e., por lo menos 20 sesiones) a fin de poder evaluar a más largo plazo el sostenimiento de los cambios logrados inicialmente.

Seguramente, éste como otros estudios deja muchas preguntas sin responder, pero no deja de ser un pequeño aporte científico para el ámbito de la psicoterapia. 


\section{REFERENCIAS}

Aspinwall, L. G. \& Taylor, S. E. (1992). Modeling cognitive adaptation: A longitudinal investigation of the impact of individual differences and coping on college adjustment and performance. Journal of Personality and Social Psychology, 61, 755-765.

Beck, A. T., Rush, J., Shaw, B. y Emery, G. (1983). Terapia cognitiva de la depresión. Bilbao: DDB.

Boland, A. \& Cappeliez, P. (1997). Optimism and neuroticism as predictors of coping and adaptation in older women. Personality and Individual Differences, 22, 909-919.

Carver, C. S., Pozo, C., Harris, S. D., Noriega, V., Scheier, M. F., Robinson, D. S., Ketcham, A. S., Moffat, F. L. \& Clark, K. C. (1993). How coping mediates the effect of optimism on distress: A study of women with early stage breast cancer. Journal of Personality and Social Psychology, 65, 375-390.

Carver, C. S., Scheier, M. F. \& Weintraub, J. K. (1989): Assessing coping strategies: A theoretically based approach. Journal of Personality and Social Psychology, 56, 267-283.

Carver, C. S. \& Gaines, J. G. (1987). Optimism, pessimism and postpartum depression. Cognitive Therapy and Research, 11, 449-462.

Carver, C. S. \& Scheier, M. F. (2002). Optimism. En Snyder y López. (Eds.): Handbook of Positive Psychology (pp. 231-243). New York: Oxford University Press.

Casullo, M. M. (2004). SCL-90-R. Adaptación UBA. CONICET. Buenos Aires: Facultad de Psicología, Universidad de Buenos Aires.

Cozzarelli, C. (1993). Personality and self-efficacy as predictors of coping with abortion. Journal of Personality and Social Psychology, 65, 12241236.

Derogatis, L. (1994). SCL-90-R. Symptom Checklist-90-R. Administration, Scoring and Procedures Manual. Minneapolis: National Computer System.

Ellis, A. y Grieger, R. (Eds.). (1981). Manual de terapia racional emotiva. Bilbao: DDB.

Fitzgerald, T. E., Tennen, H., Affleck, G. \& Pransky, G. S. (1993). The relative importance of dispositional optimism and control appraisals in quality of life after coronary artery bypass surgery. Journal of Behavioral Medicine, 16, 25-43.

Fontaine, K. R., Manstead, A. S. R. \& Wagner, H. (1993). Optimism, perceived control over stress, and coping. European Journal of Personality, 7 , 267-281.

Given, C. W., Stommel, M., Given, B., Osuch, J., Kurtz, M. E. \& Kurtz, J. C. (1993). The influence of cancer patients' symptoms and functional states on patients' depression and family caregivers' reaction and depression. Health Psychology, 12, 277-285.

Hooker, K., Monahan, D., Shifren, K. \& Hutchinson, C. (1992). Mental and physical health of spouse caregivers: The role of personality. Psychology and Aging, 7, 367-375.

Librán, Ch. E. (2002). Optimismo disposicional como predictor de estrategias de afrontamiento. Psicothema, 3, vol.14, 544-550.

Liddon, W. J. \& Jones, J. V. Jr. (2002). Terapias cognitivas con fundamento empírico: Aplicaciones actuales y futuras. México, DF.: Manual Moderno.

Litt, M. D., Tennen, H., Affleck, G. \& Klock, S. (1992). Coping and cognitive factors in adaptation to in vitro fertilization failure. Journal of Behavioral Medicine, 15, 171-187.

Scheier, M., Matthews, K., Owens, J., Magovern, G., Lefebvre, R., Abbott, R. \& Carver, C. (1989). Dispositional optimism and recovery from coronary bypass surgery: The beneficial effects on physical and psychological well-being. Journal of Personality and Social Psychology, 57, 1024-1040.

Scheier, M., Weintraub, J. \& Carver, C. S. (1986). Coping with stress: Divergent strategies of optimists and pessimists. Journal of Personality and Social Psychology, 51, 1257-1264.

Scheier, M. \& Carver, C. S. (1985). Optimism, Pessimism, and Health: Assessment and Implication of Generalized Outcomes Expectancies. Health Psychology, 4, 219-247.

Seligman, M. E. P. (2003). La Auténtica Felicidad. Buenos Aires: Vergara.

Shifren, K. \& Hooker, K. (1995). Stability and change in optimism: A study among spouse caregivers. Experimental Aging Research, 21, 59-76. 